\title{
The ability of Reciproc instruments to reach full working length without glide path preparation: A clinical retrospective study
}

\author{
Andreas Bartols ${ }^{\text {Corresp., }}{ }^{1,2}{ }^{\text {, Bernt-Peter Robra }}{ }^{3}$, Winfried Walther ${ }^{1}$ \\ ${ }^{1}$ Dental Academy for Continuing Professional Development Karlsruhe, Karlsruhe, Germany \\ 3 Institute of Social Medicine and Health Economics, Otto-von-Guericke Universität Magdeburg, Magdeburg, Germany \\ Corresponding Author: Andreas Bartols \\ Email address: andreas_bartols@azfk.de
}

Background. Reciproc instruments are the only contemporary root canal instruments where glide path preparation is no longer strictly demanded by the manufacturer. As the complete preparation of root canals is associated with success in endodontic treatment we wanted to assess the ability and find predictors for Reciproc instruments to reach full working length (RFWL) in root canals of maxillary molars in primary root canal treatment $\left(1^{\circ} \mathrm{RCTx}\right)$ and retreatment $\left(2^{\circ} \mathrm{RCTx}\right)$ cases.

Methods. This retrospective study evaluated 255 endodontic treatment cases of maxillary molars. 180 were $1^{\circ} \mathrm{RCTx}$ and $752^{\circ} \mathrm{RCTx}$. All root canals were prepared with Reciproc instruments. The groups were compared and in a binary logistic regression model predictors for RFWL were evaluated.

Results. A total of 923 root canals were treated with Reciproc without glide path preparation. This was possible in 885 cases $(95.9 \%)$. In $1^{\circ}$ RCTx cases 625 of 647 (96.6\%) canals were RFWL and in $2^{\circ} \mathrm{RCTx}$ cases 260 of $276(94.2 \%)$. In second and third mesiobuccal canals (MB2/3) 94 out of 106 (88.7\%) were RFWL with Reciproc in $1^{\circ} \mathrm{RCTx}$ and in the $2^{\circ} \mathrm{RCTx}$ treatment group 50 out of 52 cases $(96.2 \%)$. In mesiobuccal (MB1) canals " $2^{\circ} \mathrm{RCT} x$ " was identified as negative predictor for RFWL (OR 0.24 ( $\left.\mathrm{Cl} 0.08-0.77\right)$ ). In $\mathrm{MB} 2 / 3$ canals full working length was reached less often $(\mathrm{OR} 0.04(\mathrm{Cl} 0.01-0.31))$ if the tooth was obliterated and more often if MB2/3 and MB1 canals were convergent (OR 4.60 (Cl 1.07 - 19.61)).

Discussion. With Reciproc instruments the vast majority of root canals in primary treatment and retreatment cases can be prepared without glide path preparation. 
1 The ability of Reciproc instruments to reach full working length without glide path

2 preparation. A clinical retrospective study.

3 Dr. Andreas Bartols, MA ${ }^{1,2}$, Prof. Dr. Bernt-Peter Robra, $\mathrm{MPH}^{3}$, Prof. Dr. Winfried Walther ${ }^{1}$

4

51 Dental Academy for Continuing Professional Development, Karlsruhe, Germany

6 Lorenzstraße 7, 76135 Karlsruhe, Germany

72 School for Dental Medicine, Christian-Albrechts-University Kiel, Clinic for Conservative

8 Dentistry and Periodontology, Kiel

9 Arnold-Heller Str. 3 (Haus 26), 24105 Kiel, Germany

103 Institute of Social Medicine and Health Economics, University of Magdeburg, Magdeburg, 11 Germany

12 Leipziger Str. 44, 39120 Magdeburg, Germany

13

Address for correspondence: Dr. A. Bartols, Dental Academy for Continuing Professional

Development, Lorenzstraße 7, 76135 Karlsruhe, Germany

16

andreas_bartols@azfk.de

17

Phone 00497219181101 Fax 00497219181222

18

19 Running title: Reciproc treatment without glide path 


\section{Abstract}

Background. Reciproc instruments are the only contemporary root canal instruments where glide path preparation is no longer strictly demanded by the manufacturer. As the complete preparation of root canals is associated with success in endodontic treatment we wanted to assess the ability and find predictors for Reciproc instruments to reach full working length (RFWL) in root canals of maxillary molars in primary root canal treatment $\left(1^{\circ} \mathrm{RCTx}\right)$ and retreatment $\left(2^{\circ} \mathrm{RCTx}\right)$ cases.

Methods. This retrospective study evaluated 255 endodontic treatment cases of maxillary molars. 180 were $1^{\circ} \mathrm{RCTx}$ and $752^{\circ} \mathrm{RCTx}$. All root canals were prepared with Reciproc instruments. The groups were compared and in a binary logistic regression model predictors for RFWL were evaluated.

Results. A total of 923 root canals were treated with Reciproc without glide path preparation. This was possible in 885 cases $(95.9 \%)$. In $1^{\circ} \mathrm{RCTx}$ cases 625 of $647(96.6 \%)$ canals were RFWL and in $2^{\circ}$ RCTx cases 260 of $276(94.2 \%)$. In second and third mesiobuccal canals (MB2/3) 94 out of 106 (88.7\%) were RFWL with Reciproc in $1^{\circ}$ RCTx and in the $2^{\circ}$ RCTx treatment group 50 out of 52 cases $(96.2 \%)$. In mesio-buccal (MB1) canals " $2{ }^{\circ} \mathrm{RCTx}$ ” was identified as negative predictor for RFWL (OR 0.24 (CI 0.08 - 0.77)). In MB2/3 canals full working length was reached less often (OR 0.04 (CI $0.01-0.31)$ ) if the root canal was constricted and more often if MB2/3 and MB1 canals were convergent (OR 4.60 (CI 1.07 19.61)).

Discussion. With Reciproc instruments the vast majority of root canals in primary treatment and retreatment cases can be prepared without glide path preparation. 
42 Keywords: Reciproc, glide path, root canal instrumentation, endodontic retreatment. 
43

4

45

46

47

48

\section{Introduction}

Preparation of root canals in a way that it can be completely disinfected is a big challenge in endodontic treatment and retreatment. Technical difficulties are common during negotiating and preparing root canals as naturally the anatomy of root canals has a great variability (BrisenoMarroquin et al. 2015; Vertucci 1984) and especially in maxillary molars often second mesiobuccal (MB2) and sometimes even third mesiobuccal (MB3) canals are present (Kulild \& Peters 1990; Schwarze et al. 2002). These MB2/3 canals are smaller than the main canals, often covered with dentine (Zuolo et al. 2015), obliterated and curved. Moreover also the other tooth structure is often calcified (Amir et al. 2001; McCabe \& Dummer 2012). In retreatment cases more obstacles to root canal negotiation will arise, as old root canal filling materials have to be removed and procedural errors from initial treatment must be corrected (Gluskin et al. 2008) or cannot be corrected.

The latest developments in mechanical root canal preparation led to the introduction of reciprocating single file techniques (Bürklein et al. 2012; Yared 2008). Already rotary nickeltitanium (NiTi) multiple file systems enhanced the technical quality of root canal preparation (Schäfer et al. 2004). But for all rotary instruments, coronal enlargement of the canal and the preparation of a glide path was demanded (Berutti et al. 2009; West 2010). The glide path is defined as a smooth tunnel from the root canal orifice to the apical ending of the root canal (West 2010). A glide path can for example be prepared with stainless steel hand-instruments of small sizes like. ISO 06, 08 and 10 up to ISO 20. The pre-established tunnel reduces the stress on the tips of rotary root canal instruments and therefore also reduces the risk of instrument fractures.

64 With the introduction of single-file and single-use Reciproc instruments (VDW, Munich, Germany) the manufacturer claimed that the glide path preparation with these instruments 
66

67

68

69

70

71

72

generally is no longer mandatory (Yared 2011) even not in calcified MB2 canals (Yared 2013a). This was explained by the special reciprocating movement of the instruments meaning that the instruments cut dentine in a counter-clockwise (CCW) direction and are immediately released in a clockwise $(\mathrm{CW})$ motion. As the $\mathrm{CW}$ rotation is smaller than the $\mathrm{CCW}$ rotation the instrument will advance into the root canal. The releasing motion takes the stress from the instruments and prevents in combination with a new reinforced M-wire NiTi-alloy instrument fractures. Because of the reciprocating motion, the instruments should follow the naturally existent root canal path down to the apical ending of the canal (Yared 2011). That indeed a large proportion of root canals can be prepared with Reciproc without previous glide path preparation was confirmed lately (De-Deus et al. 2013; Zuolo et al. 2015).

In retreatment cases different techniques for gutta-percha removal were advocated. This involves the application of hand files, NiTi rotary files, ultrasonic (US) instruments, heat or solvents (Ferreira et al. 2001; Wilcox et al. 1987). Also special rotary NiTi retreatment files were developed to enable gutta-percha removal (Rödig et al. 2012). But these instruments have the sole purpose to efficiently remove the bulk of the old obturation materials. After that, glide path preparation remains mandatory to prepare the whole root canal (Gluskin et al. 2008). The manufacturer of Reciproc instruments claimed that also retreatment cases can be safely solved with these instruments as long as the manufacturer's instructions are followed (Yared 2013b). In several in-vitro studies this claim was confirmed (de Souza et al. 2015; Marfisi et al. 2015; Zuolo et al. 2013). But only completely filled root canals were retreated in these studies. So the question remains, if in clinical reality with incompletely filled canals with unexpected blockages, ledges and other obstacles the procedure remains as safe as believed. And, if in these situations root canal preparation can be done without glide path preparation. 
89 Therefore we analysed all our endodontic treatment cases of maxillary molars with Reciproc

90 instruments retrospectively. We sought to answer the question if in primary and also in

91 retreatment cases the technical root canal preparation can be carried out with Reciproc

92 instruments without glide path preparation to the full working length. Moreover we wanted to

93 reveal predictors for reaching or not reaching full working length. Our hypothesis was that the

94 type of treatment (primary vs. retreatment cases), constricted root canals and convergent MB2/3

95 and MB1 canals are predictors for reaching full working length with Reciproc instruments.

\section{Materials and Methods}

97 For this retrospective study, patient files from the outpatient clinic of the Dental Academy of

98 Continuing Professional Development Karlsruhe, Germany were used. Data were collected

99 without reference to patient names and completely anonymized for evaluation. Because of the

100 retrospective data collection, this study was a non-intervention clinical trial and did not interfere

101 with the psychological or physical integrity of patients. The study was conducted according to

102 the European guidelines for good clinical practice (CPMP/ICH/135/95) and according to the

103 Professional Code for Physicians of the Medical Council of the State of Baden-Württemberg.

104 The Institutional Review Board of the Baden-Württemberg Medical Council reviewed the study

105 from the ethical perspective and approved it (AZ: F-2016-031-z).

\section{Study sample}

107 For this clinical retrospective study all maxillary molars that were treated endodontically with

108 Reciproc from October 2011 to October 2015 were identified. All treatments were performed by

109 one single operator (AB) with ten years of extensive operative experience in endodontics.

110 Included were all primary root canal treatment cases $\left(1^{\circ} \mathrm{RCTx}\right)$ and all orthograde root canal 
111 retreatment cases $\left(2^{\circ} \mathrm{RCTx}\right)$. The age of the patients was 16 years or older. Excluded were teeth

112 with incomplete root development and retreatment cases with previously performed

113 apicoectomies. However, no further exclusion criteria were set regarding canal curvature,

114 radiographically narrow canals or preoperative restauration of the tooth.

115 The following information was collected from the medical records for every individual case:

116 gender of the patient, tooth number, quantity of root canals, type of treatment

$117\left(1^{\circ} \mathrm{RCTx} / 2^{\circ} \mathrm{RCTx}\right)$, contricted root canal (yes/no) separately for every canal (first mesiobuccal

118 (MB1), second/third mesiobuccal (MB2/3), distobuccal (DB) and palatal (P)), reaching full

119 working length (RFWL) or not reaching full working length (NRFWL) separately for every

120 canal, a glide path was prepared (yes/no) separately for every canal, MB2/3 and MB1 canals

121 convergent (yes/no), apical perforation (yes/no), Forfenan retreatment case (yes/no), Reciproc

122 instrument fracture (yes/no) and apicoectomy after orthograde (re-)treatment (yes/no).

\section{Treatment procedures}

124 Generally all patients were treated under local anaesthesia. All treatments were performed

125 according to the quality guidelines of the European Society of Endodontology (2006). In every

126 case a rubber dam was used and the complete treatment was done with the use of a dental

127 operating microscope (DOM) (PROergo S7, Zeiss, Jena, Germany). For all preparations a

128 VDW.SILVER Reciproc motor (VDW) in combination with a RootZX apex locator (J. Morita

129 Europe GmbH, Dietzenbach, Germany) or a VDW.GOLD Reciproc motor with integrated apex

130 locator (VDW) was used with the preset program "RECIPROC ALL". Before treatment the

131 preoperative radiographs were used to measure the approximate root canal lengths. The precise 
132 working lengths were determined during root canal preparation with the apex locator. Therefore

133 the complete root canal length was measured and $0.5 \mathrm{~mm}$ subtracted to set the WL.

134 Primary root canal treatment procedures

135 Straight line access to the root canal orifices was established with diamond coated burs and

136 Miller burs (both Komet Brasseler, Lemgo, Germany). All canal orifices including the

137 MB2/3orifices were opened with an EndoGuide bur EG7 (SS White Burs, Inc., Lakewood, New

138 Jersey, USA). In case of deeply calcified root canals the orifices were further prepared with a

139 diamond coated ultrasonic tip (3E Tip on Tigon, W\&H Bürmoos, Austria). After that a Reciproc

140 R25 was used to preflare the coronal two thirds of the root canal. The Reciproc instruments were

141 used strictly according to the manufacturers recommendations for root canal preparation without

142 glide path (Yared 2011) and according to the special recommendations for MB2 canals (Yared

143 2013a). During preparation the canals and pulp chamber were flooded with 3\% sodium

144 hypochlorite $(\mathrm{NaOCl})$. After enlargement of the coronal half of the root canal the canal was

145 scouted with a size 06 C-Pilot file (VDW). If the instrument could not be easily advanced further

146 into the canal without resistance the canal was rated as "constricted". When the R25 reached two

147 thirds of the canal length, the working length (WL) was determined using a size 10 C-Pilot file

148 (VDW) with an apex locator. When the C-Pilot file could not reach WL, the R25 was used again

149 to work in the canal. The procedure was repeated until WL could be determined passively with

150 the C-Pilot file. Afterwards the complete WL was prepared with the R25 and the preparation was

151 classified as RFWL. When the R25 did not advance further into the root canal, it was tried to

152 actively prepare a manual glide path with if necessary pre-bent $06,08,10$ and 15 C-Pilot files as

153 a matter of principle. The preparation was then classified as NRFWL with the R25. The aim was

154 in each case to gain apical patency with a $10 \mathrm{C}$-Pilot file. 
155 In cases of large root canals, e.g. palatal (P) canals, an additional R40 or R50 was used for the

156 preparation of that canal. All instruments were used in one molar and were afterwards discarded.

157 If signs of deformation were visible at the instruments, they were also immediately discarded and

158 replaced by a new instrument. All canals were further instrumented with NiTi hand files (VDW)

159 to at least ISO 35 to prevent locking of the 30 gauge Flexi-Glide Utility Tip (Vista Dental

160 Products, Racine, WI, USA) that was used for irrigation and to allow the deepest possible

161 placement of the irrigation tip towards the end of the root canal preparation. After completed

162 instrumentation all canals were irrigated with EDTA $15 \%$ and afterwards again disinfected with

$163 \mathrm{NaOCl} 3 \%$. All solutions were used with passive ultrasonic irrigation (PUI) (Irrisafe on P5

164 Newtron, Acteon Germany GmbH, Mettmann, Germany). After that, calcium hydroxide

$165\left(\mathrm{Ca}(\mathrm{OH})_{2}\right)$ was placed as root canal dressing or in case of single visit treatment a gutta-percha

166 root canal filling was placed. The detailed treatment protocol was described in an earlier

167 publication (Bartols 2013).

\section{Orthograde root canal retreatment procedures}

In retreatment cases the same instruments were used for access cavity preparation as in $1^{\circ} \mathrm{RCTx}$ cases. In case of a MB2/3 canal, the MB1 canal was prepared first with a R25 file. In cases of hard obturation materials, they were initially centre punched with a \#1 Gates bur (Komet Brasseler, Lemgo, Germany) for easier advancement of the R25. The R25 was used according to the $1^{\circ} \mathrm{RCTx}$ procedures. At first all filling material was removed. After that according to the $1^{\circ} \mathrm{RCTx}$ cases the rest of the canal was scouted with a size $06 \mathrm{C}$-Pilot file and was rated as "constricted" if the instrument could not easily advance into the apical part of the canal. All MB2/3 canals were not prepared previously and therefore constriction was determined according to $1^{\circ} \mathrm{RCTx}$ cases. When about two thirds of the WL was prepared, the root canal walls were 
178 cleaned in a brushing motion with the R25 to remove as much as possible of the old obturation

179 material. Then it was tried to introduce a $10 \mathrm{C}$-Pilot file to determine the working length with the

180 apex locator. If this was not possible, the R25 was used again, until passive negotiation with the

$18110 \mathrm{C}-\mathrm{Pilot}$ file was possible. When the WL was determined, the complete length was prepared

182 with the R25 and the preparation classified as RFWL. When a glide path had to be created, the

183 preparation was classified analogue to the $1^{\circ} \mathrm{RCTx}$ group as NRFWL. Apical patency was

184 maintained with a 10 C-Pilot file. The cleanliness of the root canal walls was inspected under

185 high magnification in the DOM. Remnants of obturation materials were removed with an Endo

186 file K15 or K25 on the P5 Newtron (both Acteon).

187 The further treatment and disinfection protocol was the same as with $1^{\circ} \mathrm{RCTx}$ group. Also for

188 retreatment cases a detailed protocol was described in an earlier publication (Bartols 2013).

\section{Statistical analyses}

190 SPSS (Version 21, Win x64, IBM, Armonk, New York, USA) was used for all statistical

191 analyses. The distributions to the different treatment groups were compared with the Pearson-

192 chi-square test. The $\alpha$-type error was set to 0.05 .

193 Binary logistic regression analyses were performed to take potential factors that affect RFWL

194 into account simultaneously. From clinical experience and theoretical considerations we

195 hypothesized that potential factors that would affect RFWL could be the type of treatment

$196\left(1^{\circ} \mathrm{RCTx}\right.$ vs. $\left.2^{\circ} \mathrm{RCTx}\right)$, constricted canals (yes/no) and in case of MB2/3, if the canal was

197 convergent with MB1. 


\section{Results}

199 We identified 255 maxillary molars that met the inclusion criteria. 180 were primary and 75

200 secondary treatment cases. Tooth and root canal distributions are summarized in Table 1.

201 We found $152(59.6 \%)$ MB2/3 canals in 255 maxillary molars. 107 (69.9\%) second mesiobuccal

202 and nine (5.9\%) third mesiobuccal canals in 153 first maxillary molars and $36(35.3 \%) \mathrm{MB} 2$

203 canals in 102 second maxillary molars. Overall 923 root canals were approached to be prepared

204 without prior glide path preparation (Table 2). This was possible in 885 cases, an overall rate of

$20595.9 \%$. In $1^{\circ} \mathrm{RCTx}$ cases 625 of $647(96.6 \%)$ canals were RFWL and in $2^{\circ} \mathrm{RCTx}$ cases 260 of

$206276(94.2 \%)$

207 There were differences in the two treatment groups $1^{\circ} \mathrm{RCTx}$ and $2^{\circ} \mathrm{RCTx}$ in the ability of

208 Reciproc instruments to reach full working length (Table 2). 175 out of 180 (97.2\%) MB1 canals

209 were prepared with Reciproc to full $\mathrm{WL}$ in $1^{\circ} \mathrm{RCTx}$ cases. In the $2^{\circ} \mathrm{RCTx}$ treatment group this

210 was possible in 67 out of 75 cases $(89.3 \%)$. The difference was statistically significant $\left(\mathrm{X}^{2}=6.81\right.$;

$211 \mathrm{p}=0.009)$. In the five NRFWL $1^{\circ} \mathrm{RCTx}$ canals it was tried to prepare a manual glide path because

212 the R25 did not advance further into the root canal. This was not possible and therefore two

213 canals were prepared incompletely, because these canals were not patent at any time during the

214 preparation procedures. In the three other canals the active glide path preparation was also not

215 possible. In these canals the further use of the R25 without glide path preparation resulted in an

216 apical perforation. In one of these roots the apical perforation was corrected with an

217 apicoectomy. In one case the tooth was extracted because the patient decided against perforation

218 repair and a surgical intervention and in the other case the patient decided to do nothing, because

219 he was clinically symptom-free. In five out of eight canals in the $2^{\circ} \mathrm{RCTx}$ group the R25 did not 
220 reach full WL and also the manual glide path preparation was not possible. These canals were

221 prepared incompletely and were never patent during preparation procedures. In the three other

222 canals the preparation without glide path ended in an apical perforation. These three apically

223 perforated roots were treated surgically.

224180 out of $180(100.0 \%)$ DB canals were fully prepared with Reciproc instruments in $1^{\circ} \mathrm{RCTx}$

225

226

227

228

229

230

231

232

233

234

235

236

237

238

239

240

cases. In the $2^{\circ} \mathrm{RCTx}$ treatment group this was possible in 69 out of 75 cases $(92.0 \%)$. The difference was statistically significant $\left(X^{2}=14.75 ; p<0.001\right)$. In the $2^{\circ} \mathrm{RCTx}$ group in four out of six cases in the DB canal a manual glide path preparation was not possible. One tooth had to be treated surgically. In the three remaining canals the use of R25 without glide path resulted in an apical perforation. Two of these cases were treated surgically.

All 255 palatal root canals were prepared to full WL regardless of the treatment group.

90 out of $101(89.1 \%) \mathrm{MB} 2 / 3$ canals were completely prepared with Reciproc in $1^{\circ} \mathrm{RCTx}$ cases. In the $2^{\circ} \mathrm{RCTx}$ treatment group this was possible in 49 out of 51 cases $(96.1 \%)$. The difference was not statistically significant $\left(\mathrm{X}^{2}=2.105 ; \mathrm{p}=0.147\right)$. In eight cases of the eleven $1^{\circ} \mathrm{RCTx}$ cases NRFWL, a manual glide path preparation was tried out. By that, seven cases were solved. In one case this was not possible and the canal was ultimately classified NRFWL. In the three remaining canals an apical perforation occurred with $\mathrm{R} 25$. In one of these teeth this was corrected with an apicoectomy. In the other cases the patients decided to do nothing, because they were clinically symptom-free. In one case a R25 file separated during MB2 preparation. The instrument was removed and the canal was prepared with a new R25 without glide path preparation. In the $2^{\circ} \mathrm{RCTx}$ group in one case the MB2 was prepared completely after manual 
241 glide path preparation. In the other case a R25 fractured. The fragment could not be removed.

242 The patient was clinically symptom-free and decided to leave the instrument in situ.

243 In the 152 teeth with an MB2/3 canal, 51 canals were classified as constricted. In twelve (23.5\%)

244 of the 51 constricted cases R25 was NRFWL, while in only one (1.0\%) of the other 101 not

245 constricted cases R25 was NRFWL. The difference was statistically significant $\left(\mathrm{X}^{2}=22.012\right.$;

$246 \mathrm{p}<0.001 ;)$.

247 Of the $152 \mathrm{MB} 2 / 3$ canals 90 were classified as convergent with MB1 and 60 as not convergent.

248 For the remaining two cases the data was missing in the medical files. In three $(3.3 \%)$ of the

249 convergent cases full WL was not reached with R25. In ten (16.7\%) of the not convergent cases

250 full WL was not reached with R25. The difference was statistically significant $\left(\mathrm{X}^{2}=8.085\right.$;

$251 \mathrm{p}=0.004)$.

252 The $2^{\circ} \mathrm{RCTx}$ group included 9 Forfenan ("Russian-Red-Cement") retreatment cases. In seven of

253 these cases R25 reached full WL. In one of the two other cases a manual glide path preparation

254 was performed ending in an apical perforation. In the other case an apical perforation resulted

255 from repeated R25 preparation without glide path. The first mentioned tooth was extracted and

256 the second tooth was treated with an apicoectomy.

257 During the preparation of 923 root canals in 255 teeth 2 instruments fractured (in $0.2 \%$ of the

258 canals and in $0.7 \%$ of the teeth) and 12 apical perforations (in $1.3 \%$ of the canals and in $4.7 \%$ of

259 the teeth) occurred.

260 The binary logistic regression models for RFWL (yes/no) are summarized in Table 3. For MB2

261 canals we found a significant influence to RFWL of the covariates "constricted root canal" and 
262 "MB2/3 and MB1 convergent" (both $\mathrm{p}<0.05$ ). The chance was smaller in constricted root

263 canals to RFWL and higher in teeth with convergent MB2/3 and MB1 canals.

264 For MB1 canals there was a significant association with the covariate "type of treatment" ( $p<$

265 0.05). In retreatment cases the chance of reaching FWL was lower than in primary treatments.

266 The covariate "constriction" was not significant in MB1 canals.

267 For DB canals we did not identify any covariates of significant influence. A regression model for

268 the P canals could not be computed because all canals reached full working length.

\section{Discussion}

270 Our retrospective clinical study shows that a large proportion of demanding root canals was

271 prepared without glide path preparation in maxillary molars. This is the case for primary root

272 canal treatments and for retreatments. In our logistic regression model we identified "convergent

$273 \mathrm{MB} 2 / 3$ and MB1 canals" as positive predictor for RFWL in MB2 canals and "root canal is

274 constricted" as negative predictor. For MB1 canals we identified " $2{ }^{\circ} \mathrm{RCTx}$ " as negative predictor

275 for RFWL.

276 Lately, a study identified "achieving patency at the root canal terminus" as an important

277 prognostic factor for improved healing of periapical lesions (Ng et al. 2011b) and for tooth

278 survival ( $\mathrm{Ng}$ et al. 2011a). Therefore it is of utmost importance, that root canals are prepared to

279 full WL, so that proper disinfection is possible afterwards. In our study RFWL automatically

280 included achieving patency with an ISO 10 C-Pilot file at the canal terminus. With rotary NiTi

281 instruments glide path preparation is necessary to prepare root canals to the apical canal terminus

282 to avoid instrument fractures and keep apical patency. A lot of instruments are available for glide

283 path preparation and the procedure often needs a lot of patience especially in cases with 
284 obliterated root canals (West 2010) and can be substantially time consuming. Moreover, if

285 manual glide path preparations are performed dentists describe higher general physical strain and

286 strain on their fingers than with Reciproc instruments (Bartols et al. 2016). If the glide path

287 preparation can be avoided this does not represent a primary biological objective, but may reduce

288 the effort for root canal preparation in the dimension of treatment time, number of instruments

289 and may reduce the physical strain for the operator.

290 It is difficult to find an appropriate comparison group for the question we sought to answer. At

291 the moment there is only one machine driven instrument system (Reciproc) whith which glide

292 path preparation is no longer strictly recommended by the manufacturer. All NiTi rotary

293 instrument systems normally need a glide path preparation to avoid instrument fractures (West

294 2010) that occur because of torsional stresses on the instrument (Berutti et al. 2009; de Oliveira

295 Alves et al. 2012). From an ethical point of view it is therefore not conceivable to compare rotary

296 instruments without glide path preparation with Reciproc in a clinical experiment head-to-head.

297 Of course such experiments are possible in in-vitro studies. But these studies do not completely

298 cover the clinical reality with a lot more practical problems especially in retreatment cases in

299 contrast to completely filled laboratory retreatment cases without obstacles like ledges,

300 blockages and other problems. So we decided to choose a retrospective study design to evaluate

301 a series of treatments that had been done anyway.

302 Overall we found a rate of $95.9 \%$ of root canals that were prepared with Reciproc instruments to

303 full WL. This is a large proportion of canals that were treated without glide path preparation. An

304 in-vitro study assessed the possibility to reach full WL with Reciproc instruments in straight and

305 moderately curved root canals of mandibular molars which was possible in $96.4 \%$ and $90.7 \%$

306 respectively (De-Deus et al. 2013). So our overall rate of RFWL lies between these values. 
307 Interestingly in the study of De-Deus et al. (2013) in 98 root canals after coronal and middle

308 third preparation of the root canals a size 10 file could not reach full WL while the repeated use

309 of the R25 led to RFWL in $67.3 \%$ of these canals. Therefore a lot of root canals in the

310 aforementioned study were prepared completely with the R25 that otherwise would have been

311 prepared incompletely.

312 There is only little information in literature on typical reference values for reaching full WL

313 during root canal treatment in a clinical situation. Two connected papers ( $\mathrm{Ng}$ et al. $2011 \mathrm{a}$; $\mathrm{Ng}$ et

314 al. 2011b) contain indirectly a proportion of root canals that were assessed as patent during root

315 canal treatment. Therefore we assume that these canals were prepared to full WL. For $1^{\circ} \mathrm{RCTx}$

316 the calculated rates from these papers are $93.5 \%$ and $94.6 \%$, respectively, and for $2^{\circ} \mathrm{RCTx} 86.0 \%$

317 and $91.1 \%$ (Ng et al. 2011a; $\mathrm{Ng}$ et al. 2011b). But there is no information about the types of root

318 canals treated. Our overall rates of RFWL with Reciproc instruments are about 2-3\% higher as

319 these values $\left(1^{\circ} \mathrm{RCTx} 96.6 \%\right.$ and $\left.2^{\circ} \mathrm{RCTx} 94.2 \%\right)$ and moreover contain very demanding

320 situations in terms of the preparation of MB2/3 canals of maxillary molars. Moreover, only

321 treatments of maxillary molars are evaluated in our study, while in the studies cited, every tooth

322 type was included. An in-vitro study evaluated the R25 for RFWL in straight and moderately

323 curved root canals in mandibular molars (De-Deus et al. 2013). For straight canals a rate of

$32496.4 \%$ for RFWL was found and a rate of $90.7 \%$ for moderately curved canals (De-Deus et al.

325 2013). In our study all (100.0\%) of the palatal canals were RFWL. All of these canals were

326 straight canals or had only slight curvatures. Definitely these canals could be prepared to full WL

327 most predictable. The buccal canals can be compared with the moderately curved canals,

328 although also severely curved canals were included in our study. 97.2\% of MB1 canals and

$329100.0 \%$ of DB canals in the $1^{\circ} \mathrm{RCTx}$ group were RFWL. So our clinical data reveal higher rates 
330

331

332

333

334

335

336

337

338

339

340

341

342

343

344

345

346

347

348

349

350

351

352

of RFWL than the in-vitro reference values. One clinical study compared the R25 and manual glide path preparation of MB2 root canals regarding RFWL in maxillary molars (Zuolo et al. 2015). Remarkably, in only $57.5 \%$ of the canals in the manual preparation group full WL was reached, while in the R25 group this was possible in $85.6 \%$ of the canals (Zuolo et al. 2015). We found that $89.1 \%$ of the MB2 canals were RFWL with R25 in the $1^{\circ} \mathrm{RCTx}$ group. So our RFWL rate is about $3 \%$ higher. Most interestingly in the $2^{\circ} \mathrm{RCTx}$ group $96.1 \%$ of the MB2 canals were RFWL with R25 in our study.

To the best knowledge of the authors there is no clinical information available for root canal retreatments performed with Reciproc instruments with the attempt to waive glide path preparation. One clinical study describes the deformation and fracture rates of Reciproc instruments also in retreatment cases (Plotino et al. 2015). But it is not clearly stated if the retreatment cases were performed without glide path preparation. Moreover this publication contains no information about the frequency of Reciproc to reach full WL. There are only invitro studies that evaluate the general possibility to use Reciproc instruments for endodontic retreatments (de Souza et al. 2015; Marfisi et al. 2015; Zuolo et al. 2013). All publications come to the conclusion, that Reciproc was the fastest system for retreatment. All studies found remaining filling material with all systems tested (de Souza et al. 2015; Marfisi et al. 2015; Zuolo et al. 2013). This is in concordance with our clinical experience, as it was necessary to control the cleanliness of the root canal walls under the DOM. All retreatment preparations were additionally fine finished with US instruments as described in the methods section to remove visible filling remnants.

The possibility to reach full WL with Reciproc in $2^{\circ} \mathrm{RCTx}$ cases was significantly lower in MB1 and $\mathrm{DB}$ canals than for $1^{\circ} \mathrm{RCTx}$ cases. The logistic regression model shows, that retreatment 
353 cases have a smaller chance for RFWL in MB1 canals, even if it is taken into account that we

354 found more constricted cases in $2^{\circ} \mathrm{RCTx}$. Additional difficulties may be pre-existent preparation

355 faults as ledges, pre-existent via falsas that were impossible to correct and previously not

356 properly approached MB1 canals that were instrumented in a wrong angle from disto-palatal

357 instead of a straight line access. Moreover it is also possible that Reciproc instruments caused a

358 deviation from the original root canal trajectory or led to complete blockages of the canal. In

359 these cases we did not find reasons for NRFWL, because it was also not possible to prepare a

360 manual glide path. Apart from that the overall frequency of $89.3 \%$ of RFWL lies well between

361 the $83.7 \%$ and $91.1 \%$ for $2^{\circ} \mathrm{RCTx}$ reported in the previously mentioned studies $(\mathrm{Ng}$ et al. $2011 \mathrm{a}$;

$362 \mathrm{Ng}$ et al. 2011b).

363 In our study in the palatal root canals it was possible to reach full WL in every case regardless of

$3641^{\circ} \mathrm{RCTx}$ or $2^{\circ} \mathrm{RCTx}$ cases. So we conclude that these canals are the safest to be prepared without

365 a glide path. We explain this by the fact that the palatal canals have less curvature, the biggest

366 sizes, surface areas and volumes (Peters et al. 2000; Wu et al. 2000) compared to mesio- and

367 distobuccal root canals.

368

369

370

371

372

373

374 RFWL in constricted root canals we can only indirectly guess that the substantially lower rate of 375 RFWL with manual preparation in MB2/3 canals in the above mentioned study of Zuolo et al.

In MB2/3 canals there was no statistical difference between the $1^{\circ} \mathrm{RCTx}$ and the $2^{\circ} \mathrm{RCTx}$ group. Interestingly the rate of RFWL was in the $1^{\circ} \mathrm{RCTx}$ group somewhat lower than in the $2^{\circ} \mathrm{RCTx}$ group. But in the $1^{\circ} \mathrm{RCTx}$ group in some cases it was possible to obtain full WL with Reciproc after manual glide path preparation. For MB2/3 preparation, our logistic regression model identified constriction of the root canal as a negative predictor for RFWL. It is remarkable, that this did not affect the other root canals. As there is no data available for the frequencies for 
376 (2015) can be partially explained by the difficult preparation of obliterated root canals. Reciproc

377 instruments overcome this problem in the way the manufacturer claims (Yared 2013a). The

378 positive predictor "convergent MB2/3 and MB1 canals" in preparation of MB2/3 canals for

379 RFWL directly correlates with our clinical experience. Normally the preparation of MB2 canals

380 is very predictable with a R25 if that canal is convergent to the MB1 canal.

381 In each of the treatment groups $1^{\circ} \mathrm{RCTx}$ and $2^{\circ} \mathrm{RCTx}$ one fracture of a $\mathrm{R} 25$ occurred. This is a

382 very low fracture rate of overall $0.2 \%$ of the canals. Both fractures occurred in MB2 canals. In

383 other studies higher fracture rates of up to $2.4 \%$ are reported with rotary NiTi instruments (Wang

384 et al. 2014; Wolcott et al. 2006). The rate of $0.2 \%$ in our study lies well in the range of the

385 clinically reported fracture rates of $0.21 \%$ (Plotino et al. 2015) and $0.56 \%$ (Zuolo et al. 2015) for

386 Reciproc.

387 In this study we observed a rate of $4.7 \%$ of apical perforations of the teeth treated. The overall

388 rate of root perforations was reported in another study that assessed procedural errors of

389 endodontic treatments with 4.5\% (Silva et al. 2012). The rate of root perforations in posterior

390 maxillary teeth was reported even higher with 5.8\%. Therefore the rates of our study are

391 comparable with these studies. A limitation of our study is the correct diagnosis of root

392 perforations. All perforations were apical perforations and were assessed with two-dimensional

393 X-rays. Therefore the information on the actual three-dimensional shape of the original trajectory

394 of the root canal is incomplete and accordingly also the assessment of the real rate of

395 perforations. In this context the original anatomy of the root canal of course will influence the

396 event of perforations. Especially in cases with abrupt apical curvatures or complete apical

397 constrictions of the canal even very flexible root canal instruments sometimes cannot follow the

398 original trajectory and may cause undetected perforations. Because this study was a retrospective 
399 study it was not possible to further evaluate the influence of the original root canal anatomy on

400 RFWL with Reciproc instruments.

401 It was possible to prepare the vast majority of endodontic primary and retreatment cases with

402 Reciproc to full working length without prior glide path preparation. Reciproc instruments

403 contribute in a highly universal way to the armamentarium of the endodontic clinician. Within

404 the limitations of this study we suggest to waive the traditional glide path preparation with

405 Reciproc instruments.

\section{References}

407 2006. Quality guidelines for endodontic treatment: consensus report of the European Society of Endodontology. International Endodontic Journal 39:921-930.

409

410

411

412

413

414

415

416

417

418

419

Amir FA, Gutmann JL, and Witherspoon DE. 2001. Calcific metamorphosis: a challenge in endodontic diagnosis and treatment. Quintessence International 32:447-455.

Bartols A. 2013. Clinical experiences with Reciproc. ENDO 7:179-187.

Bartols A, Reutter CA, Robra BP, and Walther W. 2016. Reciproc vs. hand instrumentation in dental practice: a study in routine care. PeerJ 4:e2182. 10.7717/peerj.2182

Berutti E, Cantatore G, Castellucci A, Chiandussi G, Pera F, Migliaretti G, and Pasqualini D. 2009. Use of nickel-titanium rotary PathFile to create the glide path: comparison with manual preflaring in simulated root canals. Journal of Endodontics 35:408-412. 10.1016/j.joen.2008.11.021

Briseno-Marroquin B, Paque F, Maier K, Willershausen B, and Wolf TG. 2015. Root Canal Morphology and Configuration of 179 Maxillary First Molars by Means of Micro- 
computed Tomography: An Ex Vivo Study. Journal of Endodontics 41:2008-2013.

422

423

Bürklein S, Hinschitza K, Dammaschke T, and Schäfer E. 2012. Shaping ability and cleaning effectiveness of two single-file systems in severely curved root canals of extracted teeth: Reciproc and WaveOne versus Mtwo and ProTaper. International Endodontic Journal 45:449-461. 10.1111/j.1365-2591.2011.01996.x

De-Deus G, Arruda TE, Souza EM, Neves A, Magalhaes K, Thuanne E, and Fidel RA. 2013. The ability of the Reciproc R25 instrument to reach the full root canal working length without a glide path. International Endodontic Journal 46:993-998. 10.1111/iej.12091

de Oliveira Alves V, da Silveira Bueno CE, Cunha RS, Pinheiro SL, Fontana CE, and de Martin AS. 2012. Comparison among Manual Instruments and PathFile and Mtwo Rotary Instruments to Create a Glide Path in the Root Canal Preparation of Curved Canals. Journal of Endodontics 38:117-120. http://dx.doi.org/10.1016/j.joen.2011.10.001

de Souza PF, Oliveira Goncalves LC, Franco Marques AA, Sponchiado Junior EC, Roberti Garcia Lda F, and de Carvalho FM. 2015. Root canal retreatment using reciprocating and continuous rotary nickel-titanium instruments. Eur J Dent 9:234-239. 10.4103/13057456.156834

Ferreira JJ, Rhodes JS, and Ford TR. 2001. The efficacy of gutta-percha removal using ProFiles. International Endodontic Journal 34:267-274.

Gluskin AH, Peters CI, Wong RDM, and Ruddle CJ. 2008. Retreatment of Non-Healing Endodontic Therapy and Management of Mishaps. In: Ingle JI, ed. Ingle's Endodontics 6. Hamilton, ON ;Maidenhead: BC Decker, 1088-1161. 
442 Kulild JC, and Peters DD. 1990. Incidence and configuration of canal systems in the

443

444

445

446

447

448

449

450

451

452

453

454

455

456

457

458

459

460

461

462

463

464

mesiobuccal root of maxillary first and second molars. Journal of Endodontics 16:311317.

Marfisi K, Mercade M, Plotino G, Clavel T, Duran-Sindreu F, and Roig M. 2015. Efficacy of Reciproc $((\mathrm{R}))$ and Profile $((\mathrm{R}))$ Instruments in the Removal of Gutta-Percha from Straight and Curved Root Canals ex Vivo. J Oral Maxillofac Res 6:e1. 10.5037/jomr.2015.6301

McCabe PS, and Dummer PM. 2012. Pulp canal obliteration: an endodontic diagnosis and treatment challenge. International Endodontic Journal 45:177-197. 10.1111/j.13652591.2011.01963.x

Ng YL, Mann V, and Gulabivala K. 2011a. A prospective study of the factors affecting outcomes of non-surgical root canal treatment: part 2: tooth survival. International Endodontic Journal 44:610-625. 10.1111/j.1365-2591.2011.01873.x

Ng YL, Mann V, and Gulabivala K. 2011b. A prospective study of the factors affecting outcomes of nonsurgical root canal treatment: part 1: periapical health. International Endodontic Journal 44:583-609. 10.1111/j.1365-2591.2011.01872.x

Peters OA, Laib A, Ruegsegger P, and Barbakow F. 2000. Three-dimensional analysis of root canal geometry by high-resolution computed tomography. Journal of Dental Research 79:1405-1409. 10.1177/00220345000790060901

Plotino G, Grande NM, and Porciani PF. 2015. Deformation and fracture incidence of Reciproc instruments: a clinical evaluation. International Endodontic Journal 48:199-205.

10.1111/iej.12302

Rödig T, Hausdorfer T, Konietschke F, Dullin C, Hahn W, and Hülsmann M. 2012. Efficacy of D-RaCe and ProTaper Universal Retreatment NiTi instruments and hand files in 
465

466

467

468

469

470

471

472

473

474

475

476

477

478

479

480

481

482

483

484

485

486

removing gutta-percha from curved root canals - a micro-computed tomography study. International Endodontic Journal 45:580-589. 10.1111/j.1365-2591.2012.02014.x

Schäfer E, Schulz-Bongert U, and Tulus G. 2004. Comparison of hand stainless steel and nickel titanium rotary instrumentation: a clinical study. Journal of Endodontics 30:432-435.

Schwarze T, Baethge C, Stecher T, and Geurtsen W. 2002. Identification of second canals in the mesiobuccal root of maxillary first and second molars using magnifying loupes or an operating microscope. Aust Endod J 28:57-60.

Silva JA, de Alencar AH, da Rocha SS, Lopes LG, and Estrela C. 2012. Three-dimensional image contribution for evaluation of operative procedural errors in endodontic therapy and dental implants. Brazilian Dental Journal 23:127-134.

Vertucci FJ. 1984. Root canal anatomy of the human permanent teeth. Oral Surgery, Oral Medicine, Oral Pathology 58:589-599.

Wang NN, Ge JY, Xie SJ, Chen G, and Zhu M. 2014. Analysis of Mtwo rotary instrument separation during endodontic therapy: a retrospective clinical study. Cell Biochemistry and Biophysics 70:1091-1095. 10.1007/s12013-014-0027-0

West JD. 2010. The endodontic Glidepath: "Secret to rotary safety". Dentistry Today 29:86-93.

Wilcox LR, Krell KV, Madison S, and Rittman B. 1987. Endodontic retreatment: evaluation of gutta-percha and sealer removal and canal reinstrumentation. Journal of Endodontics 13:453-457. 10.1016/S0099-2399(87)80064-X

Wolcott S, Wolcott J, Ishley D, Kennedy W, Johnson S, Minnich S, and Meyers J. 2006. Separation incidence of protaper rotary instruments: a large cohort clinical evaluation. Journal of Endodontics 32:1139-1141. 10.1016/j.joen.2006.05.015 
487 Wu MK, R'Oris A, Barkis D, and Wesselink PR. 2000. Prevalence and extent of long oval canals 488 in the apical third. Oral Surgery, Oral Medicine, Oral Pathology, Oral Radiology and Endodontics 89:739-743.

490

491

492

493

494

495

496

497

498

499

500

501

502

503

504

505

506

507

508 observations. International Endodontic Journal 41:339-344. 10.1111/j.13652591.2007.01351.x

Yared G. 2011. Canal preparation with only one reciprocating instrument without prior hand filing: A new concept. Available at http://endodonticcourses.com/cmsAdmin/uploads/RECIPROC-OL-Article.pdf.

Yared G. 2013a. Canal preparation of the MB2 canal with the R25 RECIPROC® instrument without prior hand filing or glide path. . Available at http://endodonticcourses.com/cmsAdmin/uploads/MB2 en 26-3-13.pdf.

Yared G. 2013b. Root Canal Retreatment Using the RECIPROC® System: Proficiency and Efficiency Through Simplicity. Available at http://endodonticcourses.com/cmsAdmin/uploads/Med-Artikel-RECIPROC-en_05-1112.pdf.

Zuolo AS, Mello JE, Jr., Cunha RS, Zuolo ML, and Bueno CE. 2013. Efficacy of reciprocating and rotary techniques for removing filling material during root canal retreatment. International Endodontic Journal 46:947-953. 10.1111/iej.12085

Zuolo ML, Carvalho MC, and De-Deus G. 2015. Negotiability of Second Mesiobuccal Canals in Maxillary Molars Using a Reciprocating System. Journal of Endodontics 41:1913-1917. 10.1016/j.joen.2015.08.004 


\section{Table $\mathbf{1}$ (on next page)}

Table 1. Frequencies of tooth types, root canal distributions and type of treatment. 
1 Table 1. Frequencies of tooth types, root canal distributions and type of treatment.

\begin{tabular}{|c|c|c|c|c|c|c|c|c|c|}
\hline lantity of teeth & & uantity & f root & canals $\mathrm{p}$ & $r$ to & oth & & Гуре of $t$ & eatment \\
\hline & & 3 & & 4 & & 5 & & RCTx & $2^{\circ} \mathrm{RCTx}$ \\
\hline 153 & 37 & $(24.2)$ & 107 & $(69.9)$ & 9 & $(5.9)$ & 101 & $(66.0)$ & $52 \quad(34.0)$ \\
\hline 102 & 66 & $(64.7)$ & 36 & $(35.3)$ & 0 & $(0.0)$ & 79 & $(77.5)$ & $23 \quad(22.5)$ \\
\hline 255 & 103 & $(40.4)$ & 143 & $(56.1)$ & 9 & $(3.5)$ & 180 & $(70.6)$ & $75 \quad(29.4)$ \\
\hline
\end{tabular}

2

First maxillary molar $N(\%)$

Second maxillary molar $N(\%)$

Total $N$

$31^{\circ} \mathrm{RCTx}$ - primary root canal treatment, $2^{\circ} \mathrm{RCTx}$ - orthograde endodontic retreatment 
Table 2 (on next page)

Table 2. Root canals evaluated as reaching full working length (RFWL). 
1 Table 2. Root canals evaluated as reaching full working length (RFWL).

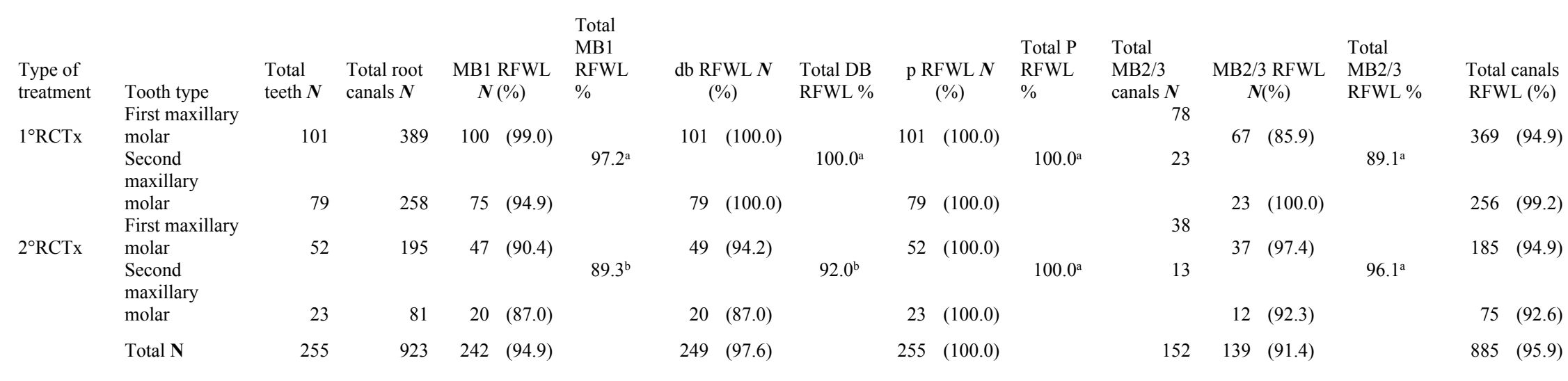

2

$31^{\circ} \mathrm{RCTx}$ - primary root canal treatment, $2^{\circ} \mathrm{RCTx}$ - orthograde endodontic retreatment, MB - mesiobuccal root canal, DB - distobuccal root canal, $\mathrm{P}$ - palatal root

4 canal. Values with different superscript letters indicate statistically significant differences in columns (Pearson-chi-square test, $p<0.05$ ). 


\section{Table 3(on next page)}

Table 3. Binary logistic regression modeling for tooth related factors affecting RFWL. 
1 Table 3. Binary logistic regression modeling for tooth related factors affecting RFWL.

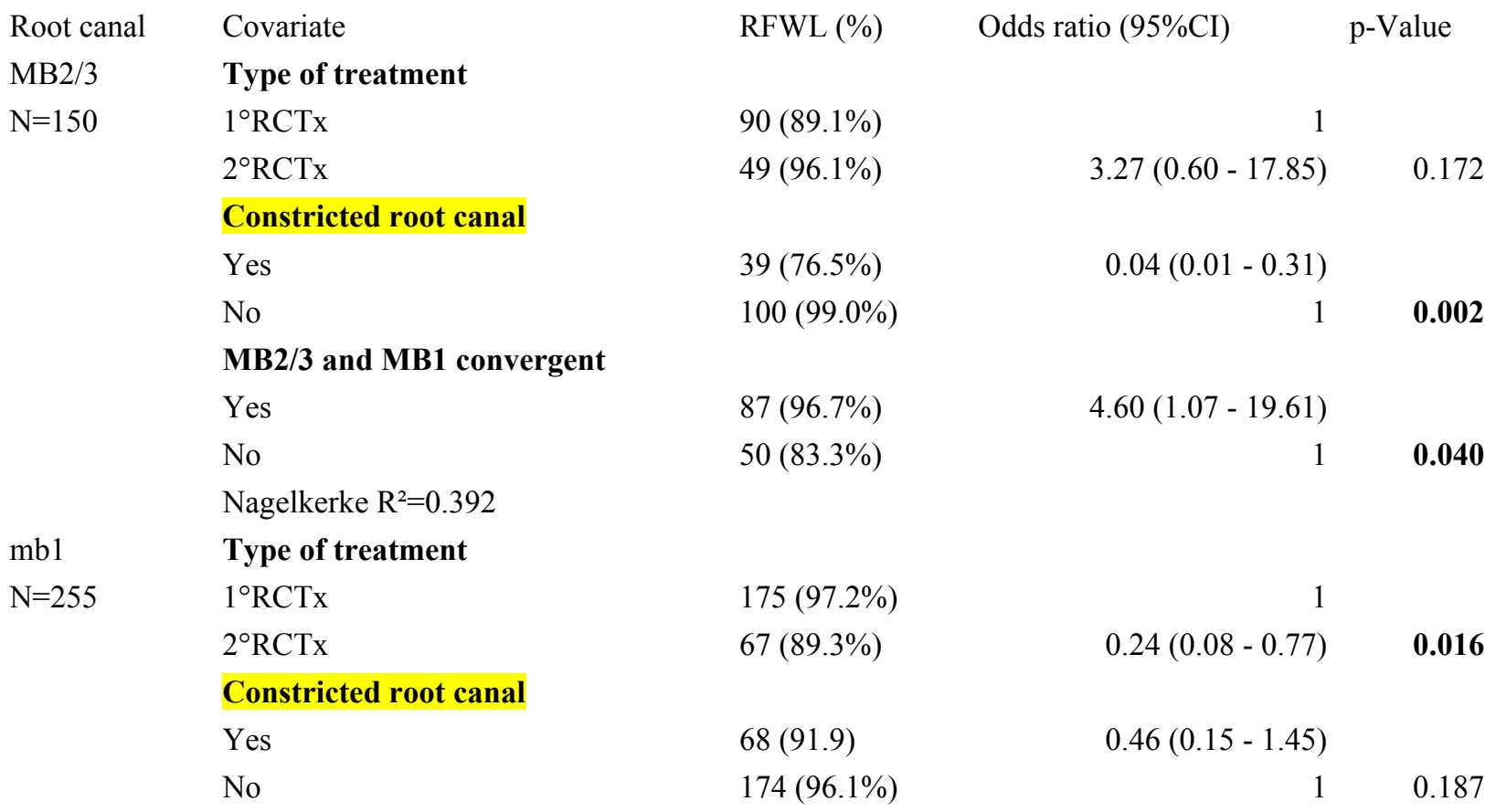

Nagelkerke $\mathrm{R}^{2}=0.091$

$2 \quad 1^{\circ} \mathrm{RCTx}$ - primary root canal treatment, $2^{\circ} \mathrm{RCTx}$ - orthograde root canal retreatment, RFWL - reaching full working

3 length. Bold $\mathrm{p}$-values indicate statistical significance of $\mathrm{p}<0.05$ in the logistic regression model 